\title{
DESENVOLVIMENTO SUSTENTÁVEL E CULTURA DE PAZ: CONCEITOS PASSÍVEIS DE UM PROCESSO DE ENSINO E APRENDIZAGEM
}

\author{
DESARROLLO SOSTENIBLE Y LA CULTURA DE PAZ: CONCEPTOS DE UN \\ PROCESO DE CALIFICACIÓN DE ENSEÑANZA Y APRENDIZAJE
}

\section{SUSTAINABLE DEVELOPMENT AND CULTURE OF PEACE: CONCEPTS OF A QUALIFYING PROCESS OF TEACHING AND LEARNING}

\author{
Rachel de Sá Barreto Luna Callou CRUZ ${ }^{1}$ \\ Verônica Salgueiro NASCIMENTO \\ Karla Jimena Araújo de Jesus SAMPAIO ${ }^{3}$ \\ Suely Salgueiro CHACON ${ }^{4}$
}

RESUMO: A presente exposição trata-se de um fragmento extraído da dissertação de mestrado em Desenvolvimento Regional Sustentável da Universidade Federal do CaririUFCA intitulada "Desenvolvimento Regional Sustentável e Violência: o papel da universidade para promoção da cultura de paz na região do Cariri”. Pretendeu-se aqui aprofundar a discussão sobre desenvolvimento sustentável e cultura de paz como peça essencial na construção do processo ensino aprendizagem nas instituições de ensino. Para tanto, são apresentados registros e reflexões acerca da temática em uma exposição de temas. Conclui-se que a busca por uma sociedade sustentável a partir da sensibilização dos indivíduos através da educação pode trazer um novo modo de ver a vida e a sociedade, de modo mais positivo, aberto e interventivo às questões conflitivas de nosso tempo, sem descuidar da complexidade dos desafios, circulando em diferentes campos de saberes, reconhecendo que não basta apenas boas intenções, mas "promover transformações necessárias e indispensáveis para que a paz seja o princípio governante de todas as relações humanas e sociais".

Palavras-chave: Desenvolvimento sustentável; cultura de paz; ensino; aprendizagem

RESUMEN: Esta exposición se encuentra en un fragmento extraído de la tesis sobre el Desarrollo Regional Sostenible, Universidad Federal de Cariri-UFCA titulado "Desarrollo

\footnotetext{
${ }^{1}$ Mestre em Desenvolvimento Regional Sustentável pela Universidade Federal do Cariri-UFCA. E-mail: rachel.callou@hotmail.com

${ }^{2}$ Doutora em Educação Brasileira pela Universidade Federal do Ceará-UFC. Professora Adjunta da UFC. Email: vesalgueiro@gmail.com

${ }^{3}$ Doutorado em Ciências pelo programa de Pós-graduação em Saúde Coletiva pela Universidade Federal de São Paulo-UNIFESP. Professora Adjunta da Universidade Regional do Cariri-URCA. E-mail: kjajs@uol.com.br

${ }^{4}$ Doutora em Desenvolvimento Sustentável pela Universidade de Brasília-UNB. Professora Adjunta da Universidade Federal do Cariri-UFCA. E-mail: suelychacon@gmail.com
} 
Regional Sustentable y la violencia: el papel de la universidad para promover una cultura de paz en el Cariri". Aquí teníamos la intención de fomentar el debate sobre el desarrollo sostenible y la cultura de la paz como una parte esencial en la construcción del proceso de aprendizaje en las instituciones educativas. Para ello, se presentan los registros y reflexiones sobre el tema de un temas de la exposición. Llegamos a la conclusión de que la búsqueda de una sociedad sostenible a partir de la conciencia de los individuos a través de la educación puede aportar una nueva forma de ver la vida y la sociedad, más positiva, abierta y de intervenir a las cuestiones conflictivas de nuestro modo de hora, sin dejar de lado la complejidad desafíos, que circulan en diferentes campos del conocimiento, reconociendo que no basta con buenas intenciones, sino "promover las transformaciones necesarias e indispensables para la paz a ser el principio rector de todas las relaciones humanas y sociales."

Palabras clave: desarrollo sostenible, la cultura de la paz, la enseñanza, el aprendizaje

\begin{abstract}
This exhibition is in an extracted fragment of the dissertation on Sustainable Regional Development, Federal University of Cariri - UFCA entitled" Regional Sustainable Development and Violence: the role of the university to promote a culture of peace in the Cariri ". Here we intended to further the discussion on sustainable development and culture of peace as an essential part in building the learning process in educational institutions. To do so, records and reflections on the theme of an exhibition themes are presented. We conclude that the search for a sustainable society through awareness of individuals through education can bring a new way of seeing life and society, more positive, open and intervening to conflicting issues of our time mode, without neglecting the complexity challenges, circulating in different fields of knowledge, recognizing that not enough good intentions but" promote transformations necessary and indispensable for peace to be the ruling principle of all human and social relations."
\end{abstract}

Keywords: sustainable development, culture of peace, teaching, learning

O conceito de sustentabilidade envolve aspectos econômicos, ambientais e sociais. A sustentabilidade social está relacionada a princípios éticos, de solidariedade e respeito aos direitos humanos, e é representada pela diminuição das desigualdades sociais, corrupção e violência. Esta temática está cada vez mais presente em nossas vidas e vem sendo discutida em teorias e práticas voltadas para o bem social (LOUETTE, 2007).

O discurso do desenvolvimento sustentável surgiu quando os cientistas manifestaram-se sobre o caminho sem volta ao qual a humanidade estava conduzindo o planeta. E, juntamente com o meio ambiente, as relações interpessoais e sociais também se degradaram ao longo dos 
anos. As transformações socioeconômicas no século $\mathrm{XX}$ acabaram por produzir uma sequência de fenômenos que não só favorecem o comportamento violento, como propiciam a sua manutenção na sociedade, com certa desordem ética, na qual nem as famílias nem os professores sabem lidar com questões de ética e moralidade (LINS et al., 2007).

Entre as décadas de 1980 e 1990, o desenvolvimento era um termo que remetia à produção de um país e a sua economia, medido através de seu Produto Interno Bruto (PIB). O


preservando o meio ambiente. Em 1987, no Relatório Brundlandt, da Comissão Mundial de Meio Ambiente e Desenvolvimento, „desenvolvimento sustentável ${ }^{\text {le }}$ foi definido como aquele que atende às necessidades do presente sem comprometer a possibilidade das futuras gerações atenderem suas próprias necessidades. Foi após a Rio 92, conferência responsável por estruturar uma responsabilidade comum sobre o planeta, principalmente por parte das nações desenvolvidas, dos governos e da opinião pública mundiais (GUIMARÃES; FONTOURA, 2012); que o foco deslocou-se do meio utilizado para os fins aos quais o desenvolvimento deve servir: o progresso global da sociedade e o seu bem-estar (UNESCO, 1999a).

O conceito de desenvolvimento sustentável, então, passou a englobar quatro dimensões básicas: a ética, que envolve a solidariedade em relação às futuras gerações; a temporal, através da precaução e planejamento em longo prazo; a social, pela diminuição das desigualdades; e a prática, que reconhece como necessária a mudança de hábitos de consumo e de comportamentos (BEZERRA; FERNANDES, 2000).

Entretanto, para Fonseca (2005), permanecem pouco discutidos os valores éticos, culturais e ambientais, que poderiam ser associados ao conceito de desenvolvimento sustentável, norteadores de uma nova racionalidade, verdadeiramente transformadora de nossos ethos (costumes) e de nossas práxis (ações). Afinal, o objetivo não deve ser tão somente o desenvolvimento sustentável, mas uma sociedade sustentável, identificada por uma cultura de paz, baseada em valores, atitudes, comportamentos e vivências que reforçam a nãoviolência e o respeito à liberdade e aos direitos fundamentais dos indivíduos (UNESCO, 1999b).

Nesse contexto, discutir sobre meio ambiente, desenvolvimento sustentável e sustentabilidade, é discutir sobre o papel do ser humano, da natureza, das relações sociais, ou seja, a vida que circula no planeta terra, casa comum da humanidade. Isso pode ocorrer a 
partir de intervenções que possibilitem uma consciência crítica e ações voltadas para uma cultura de paz que propõe uma ética de solidariedade que pode ser construída através do desenvolvimento sustentável a partir da co-responsabilidade. Meio ambiente equilibrado é resultado do desenvolvimento sustentável, direção possível de um produto final que é a cultura da paz através de um processo de educação.

As definições mais usadas de desenvolvimento sustentável o vinculam ao desenvolvimento social, econômico e ambiental, no sentido de melhorar as condições de vida da população dentro dos limites da capacidade de sustento dos ecossistemas. A vinculação do desenvolvimento social com o meio ambiente é necessária para que se possa alcançar o desenvolvimento sustentável, que articula os elementos ambientais, econômicos e sociais.

Atualmente tem se falado muito de sustentabilidade que nada mais é do que "uma adequação entre as exigências ambientais e as necessidades do desenvolvimento" (IBAMA, 1999, p. 31). Garantir a sustentabilidade ambiental constitui-se um dos grandes objetivos do Plano Nacional das Nações Unidas para o Desenvolvimento (PNUD), pois segundo as informações desta instituição, um bilhão de pessoas no planeta ainda não tem acesso à água potável, muito menos saneamento básico. E estes dois fatores são essenciais para a qualidade de vida da população. Observa-se que sem a adoção de políticas e programas ambientais, pouco se fará para a conquista da melhoria de vida das pessoas. O desenvolvimento sustentável, para Sachs (1993), tem por objetivo alcançar um crescimento econômico e social que não dilapide o patrimônio natural das nações sem perturbar os equilíbrios ecológicos.

Uma das perspectivas da sustentabilidade é o fato de que o desenvolvimento, ao preocupar-se com a geração de riquezas, tenha por objetivo distribuí-las para melhorar a qualidade de vida da população e consequentemente a qualidade ambiental do planeta. $\mathrm{O}$ desenvolvimento sustentável não desconsidera a igualdade, a justiça social e o fortalecimento da cidadania. Ele preocupa-se com a qualidade de vida e bem-estar da sociedade, ao mesmo tempo em que provoca em cada ser humano, o sentimento de pertencimento e cidadania. Neste sentido, o desenvolvimento sustentável busca atender às necessidades presentes da população planetária, mas na perspectiva do cuidado de forma que as ações do cidadão de hoje, não comprometam as gerações futuras (OLIVEIRA, 2007).

Há no contexto atual, uma necessidade da sociedade organizar-se de forma diferente com a natureza e com seus modos de produção, levando em consideração a sustentabilidade que se refere às maneiras de pensar o mundo e às formas de prática pessoal e social onde os 
indivíduos tenham ações norteadas por princípios éticos e comunidades com compromissos coletivos baseados na tolerância e igualdade, sistemas sociais e instituições participativas e práticas ambientais que valorizem e sustentem processos ecológicos de apoio à vida

(UNESCO, 2005, p.30). Neste sentido, é necessário "tomar partido por valores que possibilitem a mudança pessoal e social" (RAYO, 2004, p. 104-105).

Para Boff (2008), a sociedade vive uma crise civilizacional, e a mesma nos leva a um novo paradigma de convivência que funde uma relação benfazeja com a terra, inaugurando um tipo de respeito e cuidado e preservação de tudo o que existe e vive. Alternativas que representam uma esperança. $\mathrm{O}$ autor fala de um Ethos no sentido originário de casa humana, ou melhor dizendo; "aquela porção do mundo que reservamos para organizar, cuidar e fazer nosso habitat"(BOFF, 2008, p. 27).

O cuidado é algo que está na raiz primeira do ser humano, mas é preciso saber cuidar, a necessidade do cuidado tem que passar pelo coração e não apenas pela consciência, pois é o sentimento, aquilo que passou por uma emoção que provoca cuidado e deixa marcas profundas que podem permanecer definitivamente. Neste sentido, cada ser humano precisa desenvolver e/ou fortalecer sua dimensão anima, o que significa conceder direito de cidadania à nossa capacidade de sentir o outro, de guiar-se mais pela lógica do coração e do cuidado, da gentileza, da cordialidade, do que da lógica da conquista do uso utilitário das coisas, a partir de um relacionamento de humanos entre si e com a natureza articulando uma troca de saberes (OLIVEIRA, 2007).

No que concerne à cultura de paz, esta vem sendo considerada no mundo acadêmico com um paradigma emergente. Entre os diferentes conceitos de cultura de paz, há o que reconhece a paz, como algo que tem raízes sociais, econômicas e políticas a partir de uma base cultural.

De acordo com Mayor (2000) o conceito de paz relaciona-se à justiça social:

Não pode haver paz sustentável sem desenvolvimento sustentável. Não pode haver desenvolvimento sem educação ao longo da vida. Não pode haver desenvolvimento sem democracia, sem uma distribuição mais equitativa dos recursos, sem a eliminação das disparidades que separam os países mais avançados daqueles menos desenvolvidos (MAYOR, 2000).

Na perspectiva de Mayor (2000), a cultura da paz nos leva a uma ação a partir de uma relação entre paz, desenvolvimento e democracia. Neste sentido, entendemos a paz como 
algo social, uma vez que ela luta contra exclusão e busca a equidade social e a diversidade cultural. Situar-nos diante da dimensão da paz tem se constituído uma tarefa difícil em tempos de guerra, de crescente aumento das desigualdades, de corrupção, de injustiça social, entre outros. A paz vem emergindo cada vez mais como um clamor universal. "A degradação do meio ambiente, da economia e da cultura fazem a humanidade experimentar o problema do debilitamento do ser e levantar uma agenda ética comum" (GUIMARÃES, 2006. p. 01). Não é suficiente desejar a paz, é preciso antes de tudo se colocar numa posição de buscar estratégias concretas de construir a paz, e cada ser humano pode junto com o desejo de paz, se perguntar como se pode colaborar com a construção de uma cultura de paz. A consciência da importância da não violência está crescendo cada vez mais e no "horizonte do mundo, desenha-se um novo senso comum emancipatório e uma prática social eminentemente nãoviolenta" (GUIMARÃES, 2006, p. 02).

Adams (2003), um dos responsáveis pelo desenvolvimento do programa cultura de paz da Unesco, relata que a humanidade, em se tratando de desenvolvimento, encontra-se num processo de cruzamento de fronteiras onde a transformação de uma cultura da guerra para uma cultura de paz seja talvez a mais radical e abrangente, que qualquer mudança anterior da história da humanidade. Pode-se dizer que a construção de uma sociedade sustentável, exige um trabalho gradativo de enfrentamento e a prevenção da violência inclui a busca constante de uma cultura de paz, isto é "modos de vida, padrões, crenças, valores e comportamentos, bem como arranjos institucionais que promovem o bem-estar, bem como a igualdade que inclui o reconhecimento das diferenças" (MILANI, 2003, P. 35).

A prevenção e o enfrentamento da violência deve levar em conta o resgate de valores construídos historicamente, mas que no contexto atual, diante da velocidade das mudanças, estão gradativamente se perdendo. É necessário que as instituições de educação trabalhem em prol de uma cidadania que reconheça as diferenças, bem como a singularidade dos seres humanos. Outro aspecto muito importante a ser considerado, é a inclusão que provoca no educador um novo olhar, que é de ressignificação da identidade do aluno. Mas esse novo olhar suscita crises nos contextos educacionais, principalmente onde se trabalha ainda com uma educação bancária, que segundo Paulo Freire (2005) é aquela que tem por finalidade manter a divisão entre os que sabem e os que não sabem, entre os oprimidos e opressores, negando a dialogicidade e a relação dialógico-dialética entre educador e educando, onde ambos aprendem juntos. 
Na educação bancária predominam os sistemas de ensino onde se buscam soluções para atender aos apelos desse momento contemporâneo que pede por uma educação que vá além da reprodução do conhecimento, onde os ambientes educacionais se criam para alguns e não para todos de forma incondicional (FREIRE, 2005).

A educação tem como finalidade o estímulo à criação cultural e o desenvolvimento do espírito científico e do pensamento reflexivo, destacando a formação de diplomados aptos para a inserção em setores profissionais e para a participação no desenvolvimento da sociedade brasileira, colaborando para sua formação contínua (BRASIL, 1996).

O grande desafio dos professores e instituições educacionais é formar profissionais críticos-reflexivos no intuito de tornarem-se agentes inovadores e transformadores da realidade, inseridos na busca por ações e estratégias integrativas na solução da problemática ambiental e social, pois a enfermagem deve atender as necessidades presentes, mas sem esquecer as gerações futuras, defendendo o bem estar humano e ambiental.

Desse modo, instiga-se o desafio da busca por uma atuação nos processos de formação, com educação permanente da temática ,sustentabilidade .

A inclusão social aqui referida significa desenvolver processos que assegurem que todos os discentes tenham acesso a todas as oportunidades oferecidas pela escola ou universidade em que estudam. Conforme Mitller (2003, p 26), "No contexto da educação, a reestruturação das instituições baseadas em diretrizes inclusivas é reflexo de um modelo de sociedade em ação". Essa inclusão requer que todo o corpo educacional tenha direito à preparação apropriada no decorrer de seu exercício profissional, porque a inclusão requer mudanças na forma de pensar e agir, nos valores para os profissionais da educação e para a sociedade em geral, que por sua vez está repleta de desigualdades, que se refletem diariamente no sistema educacional.

Paulo Freire, ao referir-se ao trabalho pedagógico, sugeriu que professores e alunos fossem parceiros nas conquistas do conhecimento que emancipa a pessoa, através do entusiasmo, da alegria de ensinar e de aprender e da partilha da descoberta da curiosidade, cultivados numa relação pedagógica, onde o professor reviva a cada momento sua prática docente (FREIRE, 2005, p.103).

O campo educacional configura-se como campo propício para intervenção nas dimensões do Estado e da vida social, que se coloca como estratégica na sociedade 
contemporânea, tanto na ampliação e integração das lutas sociais como com relação ao acesso aos direitos e ao enfrentamento das desigualdades (ALMEIDA, 2000, p. 24).

Nesse sentido, sendo a paz e violência conceitos passíveis de um processo de ensino e aprendizagem, os educadores devem se ocupar diretamente de seu processo de problematização. É necessário desnaturalizar os conceitos de paz e de violência e, consequentemente, implementar a cultura de paz. Estes princípios devem seguir o caminho educativo, devendo passar necessariamente pelas instituições de ensino (NASCIMENTO, 2009).

Há três formas de enfrentamento da violência: através da repressão; a estrutura social e econômica como a solução para todos os problemas; e a terceira diz respeito à implementação de uma cultura de paz (MILANI, 2003). Esta última é alicerçada por Freire (2005) ao afirmar que promover o processo de conscientização é um passo importante para a construção da paz, através da ampliação da leitura de mundo no qual o sujeito encontra-se inserido com vistas à transformação de ambos.

A busca por uma sociedade sustentável a partir da sensibilização dos indivíduos através da educação pode trazer um novo modo de ver a vida, a sociedade, modo mais positivo, aberto e interventivo às questões conflitivas de nosso tempo, sem descuidar da complexidade dos desafios, circulando em diferentes campos de saberes, reconhecendo que não basta apenas boas intenções, mas "promover transformações necessárias e indispensáveis para que a paz seja o princípio governante de todas as relações humanas e sociais” (MILANI, 2003, p. $31)$.

Tanto a cultura da paz, quanto o desenvolvimento sustentável, tratam do "respeito ao próximo, incluindo as gerações presentes e as gerações futuras, à diferença e à diversidade" (UNESCO, 2005, p. 18), fundamentalmente como valores éticos centrais.

A temática é sedutora para quem a estuda, no entanto, pode ser desestimulante para quem tenta enfrentá-la na prática. A impressão que se tem é a de que, para se fazer algo que realmente contribua para diminuir a violência, são necessários altos investimentos econômicos e políticos. A tendência é esperarmos que as contribuições para enfrentar o problema venham de uma instância superior, com a qual não temos contato e, na verdade, nem sequer conhecemos ou sabemos quem é. Os profissionais, em geral, tendem a delegar esta problemática a entidades abstratas e impessoais, eximindo-se de qualquer responsabilidade que possa, eventualmente, ocorrer em sua prática docente e/ou assistencial. 
A complexidade dos fatores que circundam a violência exige o envolvimento de várias áreas do conhecimento para a resolução do problema. Os casos precisam ser vislumbrados de forma transdisciplinar, pois o conhecimento e a capacidade de lidar com problemas específicos não devem ser entendidos como propriedades exclusivas de nenhuma disciplina ou graduação.

\section{REFERÊNCIAS}

ADAMS, David. História dos primórdios da cultura da paz. In. Memórias pessoais, agosto, 2003. Disponível em: www.comitepaz.org.br. Acessado em 08/12/2012.

ALMEIDA, Ney Luiz Teixeira. O Serviço Social na educação. Revista Inscrita, Mato Grosso, p. 19-24, 2000.

BEZERRA, M. C. L.; FERNANDES, M. A. (Coord.). Cidades sustentáveis: subsídios à elaboração da Agenda 21 brasileira. Brasília, DF: Ministério do Meio Ambiente, 2000.

BOFF, Leonardo. Saber cuidar: ética do humano - compaixão pela terra. 15. ed. Petrópolis, Rio de Janeiro: Vozes, 2008.

BRASIL. Ministério de Educação e Cultura. LDB - Lei no 9394/96, de 20 de dezembro de 1996. Estabelece as diretrizes e bases da Educação Nacional. Brasília: MEC, 1996.

FONSECA, D. P. R. Discutindo os termos de uma equação de congruência: cultura e desenvolvimento sustentável. In: GOMES, M. F. C. M.; PELEGRINO, A. I. C. (Org.).

Política de habitação popular e trabalho social. Rio de Janeiro: DP\&A, v. 1, p. 115-128, 2005.

FREIRE. Pedagogia da Autonomia: saberes necessários à prática educativa. 31.ed, São Paulo: Paz e Terra, 2005.

GUIMARÃES, R.P; FONTOURA, Y.S.R. Rio+20 ou Rio-20? Crônica de um fracasso anunciado. Ambient. soc., São Paulo, v. 15, n.3, p. p. 19-39, set./dez.2012.

IBAMA: Educação para um futuro sustentável: uma visão transdisciplinar para ações compartilhadas./UNESCO. Brasília: Edições IBAMA, 1999.

LINS, M . J. S. da C. et al. Avaliação da aprendizagem de ética em curso de formação de professores de Ensino Fundamental. Ensaio: Avaliação e Políticas Públicas em Educação, Rio de Janeiro, v. 15, n. 55, p. 255-276, abr./jun. 2007. 
LOUETTE, A. (Org.). Gestão do conhecimento - compêndio para a sustentabilidade: ferramentas de gestão de responsabilidade socioambiental. São Paulo: Antakarana Cultura Arte Ciência: Willis Harman House, 2007.

MAYOR, Frederico. Nutrindo uma cultura de paz. In. Comitê Paulista para a década da cultura de paz: um programa UNESCO: 2000-2010. Disponível em: www.comitepaz.org.br. Acessado em: 08 dez.2012.

MILANI, Faizi. Cultura de paz: estratégias, mapas e bússolas. Salvador: INPAZ, 2003.

MITTLER, Peter. Educação inclusiva: contextos sociais. Porto Alegre: Artmed, 2003.

NASCIMENTO, V.S. A Promoção da Cultura de Paz nas Escolas: a ótica das juventudes. Dissertação [Doutorado em Educação Brasileira], Universidade Federal do Ceará, Fortaleza, 2009.143p

OLIVEIRA, Simone Barros de Oliveira. Desenvolvimento Sustentável e Cultura de Paz: Desafios para o Serviço Social. [Mestrado], PPGSS/FSS/PUCRS. Porto Alegre, 2007. 140p.

RAYO, José Tuvilla. Educação em direitos humanos: ruma a uma perspectivas global. Porto Alegre: Artmed, 2004.

SACHS, Ignacy. Estratégias de transição para o século XXI: desenvolvimento e meio ambiente. São Paulo: Stúdio Nobel, 1993.

UNESCO. Educação para um futuro sustentável: uma visão transdisciplinar para ações compartilhadas. Brasília, DF: IBAMA, 1999a.

UNESCO. Cooperative peace in Southeast Asia. Jakarta: United Nations Educational, Scientific and Cultural Organization, 1999b.

UNESCO. Década das Nações Unidas para o desenvolvimento sustentável: 2005-214: Documento final do plano internacional de implementação. Brasília: UNESCO, OREALC, 2005. 